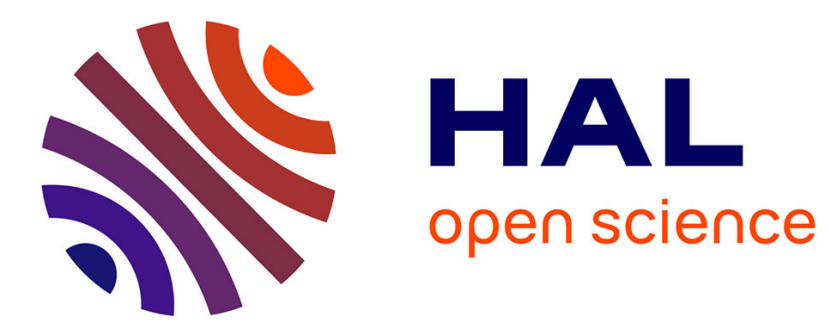

\title{
Improving 3-dimensional turbo codes using 3GPP2 interleavers
}

\author{
Dhouha Kbaier, Catherine Douillard, Sylvie Kerouedan
}

\section{To cite this version:}

Dhouha Kbaier, Catherine Douillard, Sylvie Kerouedan. Improving 3-dimensional turbo codes using 3GPP2 interleavers. ComNet'09: 1st International Conference on Communications and Networking, Nov 2009, Hammamet, Tunisia. hal-00623610

\section{HAL Id: hal-00623610 https://hal.science/hal-00623610}

Submitted on 14 Sep 2011

HAL is a multi-disciplinary open access archive for the deposit and dissemination of scientific research documents, whether they are published or not. The documents may come from teaching and research institutions in France or abroad, or from public or private research centers.
L'archive ouverte pluridisciplinaire HAL, est destinée au dépôt et à la diffusion de documents scientifiques de niveau recherche, publiés ou non, émanant des établissements d'enseignement et de recherche français ou étrangers, des laboratoires publics ou privés. 


\title{
Improving 3-Dimensional Turbo Codes Using 3GPP2 Interleavers
}

\author{
D. Kbaier Ben Ismail, C. Douillard and S. Kerouédan \\ Institut Telecom, Telecom Bretagne. UMR CNRS 3192 Lab-STICC. Technopôle Brest Iroise CS 8381829238 \\ Brest Cedex 3 \\ Université européenne de Bretagne, France \\ Email: $\{$ dhouha.kbaier,catherine.douillard,sylvie.kerouedan $\} @$ telecom-bretagne.eu
}

\begin{abstract}
This paper deals with the performance improvement of a 3-dimensional turbo code based on the partial concatenation of the 3GPP2 code with a rate-1 post-encoder. First, we optimize the distance spectrum of the 3-dimensional 3GPP2 turbo code by means of the adoption of a non regular post-encoding pattern. This allows us to increase the minimum Hamming distance and thereby to improve the performance at very low error rates. Then, we propose a time varying construction of the postencoded parity in order to reduce the observable loss of convergence at high error rates. The different improvement stages are illustrated with simulation results, asymptotical bounds and EXIT charts.
\end{abstract}

Keywords- turbo codes, iterative decoding, 3dimensional turbo codes, 3GPP2, convergence threshold, time varying trellis.

\section{INTRODUCTION}

Turbo codes (TCs) [1] have been adopted in various communication standards [2-5] due to their near-capacity performance and low decoding complexity. But they suffer from a flattening around $10^{-5}$ of Frame Error Rate (FER). In future system generations, lower error rates will be required to open the way to real time and more demanding applications, such as TV broadcasting or videoconferencing.

In [6], a 3-dimensional turbo code (3D TC) was introduced, combining both parallel and serial concatenation. It is simply derived from the classical turbo code (TC) by concatenating a rate-1 post-encoder at its output, which encodes only a fraction $\lambda$ of the parity bits from the upper and lower constituent encoders. The fraction $1-\lambda$ of parity bits which is not re-encoded is directly sent to the channel or punctured to achieve the desired code rate. The 3D TC improves performance in the error floor compared to the $\mathrm{TC}$, at the expense of a slight loss in convergence and a small increase in complexity [6].

In our work, we focused on the 3GPP 2 code, an 8-state binary turbo code, used in the third generation (3G) mobile phone communication systems [5]. The 3GPP2 turbo code is built from the parallel concatenation of two 8-state Recursive Systematic Convolutional (RSC) codes, with generator polynomials 13 (recursivity) and 15 (redundancy). The overall code rate before puncturing is $1 / 3$.

This paper is organised as follows. In Section II, we present the 3D TC structure. The decoding process is also briefly discussed in the same section. Then, in Section III, we introduce a method to optimize the 3D 3GPP2 TC in order to increase even more the minimum Hamming distance. Several upper bounds on the minimum distance of 3D binary turbo codes with 8-state upper and lower constituent encoders and 3GPP2 interleavers are presented. Furthermore, we discuss convergence issues in Section IV; and we introduce time varying 3-dimentional 3GPP2 turbo codes as an alternative to reduce the observable loss of convergence. Finally, section V draws some conclusions.

\section{CODING SCHEME}

\section{A. Encoding Structure}

A block diagram of the 3D turbo encoder is depicted in Fig. 1. In general, the post-encoder must be simple to limit the complexity increase of the corresponding decoder, and must not exhibit too much error amplification (see [6] for details), to prevent from a high loss in convergence. A 4-state binary convolutional encoder is used in practice.

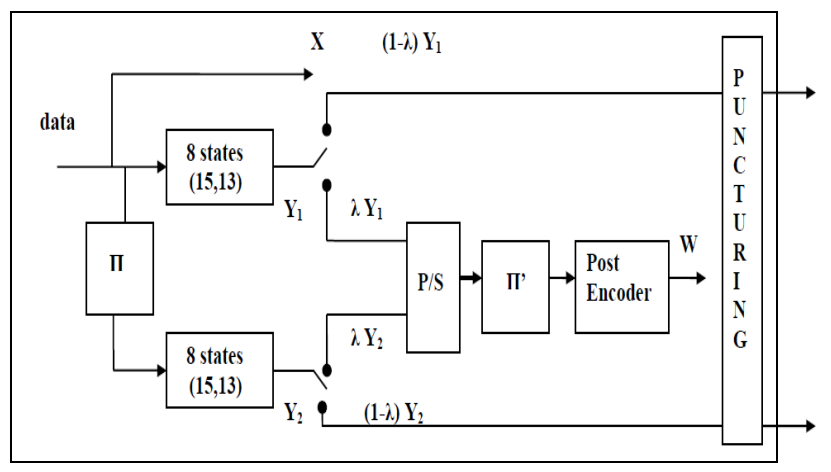

Figure 1. 3D turbo encoder structure. A fraction $\lambda$ of the parity bits from both component encoders are grouped by a P/S multiplexer, permuted by the permutation $\Pi$ ', and encoded by a rate- 1 post-encoder. 
Two linear RSC codes having memory 2 are given in Fig. 2. These possible candidates are simple. Besides, if the convolutional code is circular, it prevents from any side effects as the initial state and the final state of the post encoder are always the same [6].

The code (a) in Fig. 2 has a corresponding decoder which triples the number of errors of its input at the first step of the iterative process. Whereas the decoder of code (b) will only double the number of errors at the first iteration. Therefore, from a convergence point of view, code (b) has been selected to be the post-encoder in our simulations.

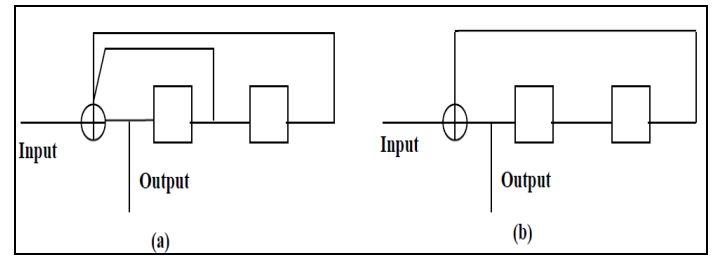

Figure 2. Possible linear post-encoder candidates with memory 2.

If we choose a large value of $\lambda$, the minimum distance is significantly increased. However, the performance in the waterfall region is degraded [6]. Thus, there is a trade-off between performance in the waterfall and error floor regions.

Usually, very simple regular permeability patterns are applied. For instance, if $\lambda=1 / 4$ the bits to be post-encoded are chosen in the regular basis $\{1000\}$ for both the upper and the lower encoders.

\section{B. Decoding Process}

The classical turbo principle is used to decode the 3D turbo code. We have three decoders corresponding to the three constituent encoders; and all of them exchange extrinsic information.

First, the 4-state SISO pre-decoder is activated to feed the two 8-state decoders with extrinsic information about the post-encoded parity bits. The two 8-state decoders exchange extrinsic information about the systematic bits, as for the classical turbo procedure. They also provide the pre-decoder with extrinsic information about the post-encoded parity bits. The decoding process continues iteratively until all constituent decoders have converged, or a maximum number of iterations has been performed.

Compared to a classical turbo decoder, the additional complexity is mainly due to the implementation of the binary 4-state decoder but also to the calculation of the extrinsic information about the post-encoded parity bits.
III. INCREASING THE MINIMUM HAMMING DISTANCE OF THE 3-DIMENSIONAL 3GPP2 TURBO CODE

\section{A. Performance of the 3-Dimensional 3GPP2 Turbo Code without Optimization}

The Frame Error Rate (FER) performance of the 3D 3GPP2 TC has been simulated with $\lambda=1 / 8$ and $\lambda=1 / 4$. Then one bit out of eight (respectively one bit out of four) is regularly picked from each of the parity streams starting with the first bit from each stream.

Fig. 3 shows that the use of 3D TC results in an increase in the minimum Hamming distance $\left(d_{\min }\right)$ by more than $28 \%$ for code rate $R=1 / 2, \lambda=1 / 8$ and $k=1530$ bits (where $\mathrm{k}$ is the number of data bits), compared to the standardized 3GPP2 turbo code. For $k=1146$ bits, code rate $R=2 / 3$ and $\lambda=1 / 4$, the increase in $d_{\text {min }}$ is even larger, more than $70 \%$, (not represented in the figure). It is possible to increase even more this gain in distance by using an irregular pattern of permeability as explained in the following paragraph.

\section{B. Optimization Method}

To obtain the distance spectrum of the 3D 3GPP2 TC, we apply the all-zero iterative decoding algorithm [7]: this technique is based on the transmission of an all-zero sequence corrupted by an impulsive noise. It allows us to determine low weight codewords and to estimate their multiplicity.

We have observed in the distance spectrum of the 3D 3GPP2 TC that the first terms have a low multiplicity. The idea is to eliminate the corresponding codewords in order to increase the minimum distance $d_{\text {min }}$. Therefore, we have modified the pattern of post-encoding, which is no more regular, to generate more ones in the codeword with the lowest weight.

\section{Optimization Results for $k=1530$}

Table I and Table II provide the first terms of the distance spectrum obtained for respectively $k=1530$ bits and $k=1146$ bits.

TABLE I. FIRST TERMS OF THE DISTANCE SPECTRUM FOR A 3 D TC WHERE $K=1530$ BITS, $R=1 / 2$ AND $\Lambda=1 / 8$.

\begin{tabular}{|l|l|l|l|l|l|l|l|}
\hline Distance & 18 & 20 & 21 & 22 & 23 & 25 & 29 \\
\hline Multiplicity & 1 & 1 & 4 & 2 & 2 & 1 & 1 \\
\hline
\end{tabular}

Table I shows that there is only one codeword with weight 18 . We have changed the pattern of post-encoding to generate more ones and to eliminate this codeword.

The codeword with weight 18 was eliminated. But it was not possible to eliminate the codeword with weight 20 , since there are many low weight codewords in the distance spectrum. So, the 
minimum distance was finally increased by 2 , and the new distance of the optimized 3D 3GPP2 TC is 20.

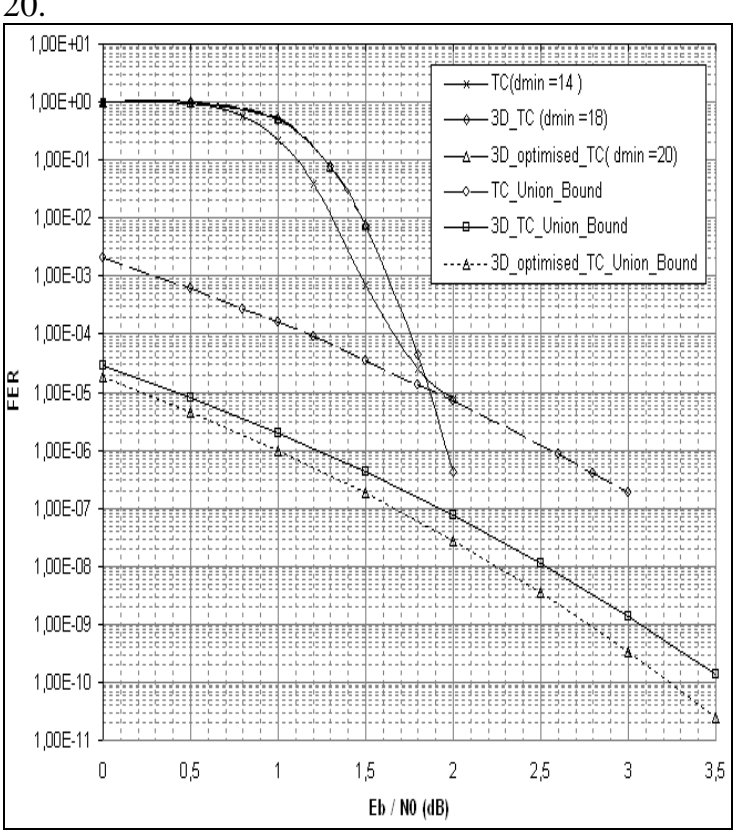

Figure 3. $\quad$ FER performance of the optimized 3D TC with $\lambda=1 / 8$ for $k=1530$ bits, $R=1 / 2$ and comparison with the 3D TC and 3GPP2 standardized TC. All simulations used the Max-LogMAP algorithm with 10 iterations.

Now compared to the standardized 3GPP2 TC, the optimization of the post-encoding pattern resulted in an increase in $d_{\min }$ by more than $42 \%$ for code rate $R=1 / 2, \lambda=1 / 8$ and $k=1530$ bits, which provides a gain of 2.5 decades in the error floor as shown in Fig. 3 with the asymptotical bounds. In fact, on the Gaussian channel, the Frame Error Rate can be upperbounded by the union bound:

$$
F E R \leq \frac{1}{2} \sum_{d \geq d_{\min }} n(d) \operatorname{erfc}\left(R d \frac{E_{b}}{N_{0}}\right)
$$

Where $n(d)$ is the code multiplicity (number of codewords with weight $d$ ), and $\operatorname{erfc}(\mathrm{x})$ is the complementary error function.

\section{Optimization Results for $k=1146$}

The same kind of optimization was performed with another frame length, $k=1146$.

TABLE II. FIRST TERMS OF THE DISTANCE SPECTRUM FOR A 3 D TC WHERE $K=1146$ BITS, $R=2 / 3$ AND $\Lambda=1 / 4$.

\begin{tabular}{|l|l|l|l|l|}
\hline Distance & 12 & 15 & 47 & 48 \\
\hline Multiplicity & 1 & 3 & 2 & 1 \\
\hline
\end{tabular}

Table II shows that there is only one codeword with weight 12 . In this codeword, we have noticed that there are only ones in the systematic part for the systematic bits at addresses $\{586,587,591$, $650,651,655,763,764,768,1019,1020,1024\}$, and the corresponding parity bits do not benefit from the post encoding which occurs regularly for the bits which address modulo 4 is equal to 1 . To optimize the 3D 3GPP2 TC, we have slightly modified the permeability pattern in order to postcode the bits at addresses $\{585,587,650,651$, 763 and 764$\}$ instead of $\{9,101,581,925,1029$ and 1133$\}$. Also, we have chosen to spread our modifications on all the length of the frame, not to discriminate a given region.

The idea was easier to implement, compared with $k=1530$ bits, since there were few low weight codewords in the spectrum. That's why we have succeeded in eliminating the codewords with lower weights ((i.e., 12 and 15) at once.

The new minimum distance of the optimized 3D-TC is 33 (see Table III). This value has to be compared to 7 which is the distance of the standardized 3GPP2 TC. The use of optimized permeability patterns resulted in a huge increase in $d_{\text {min }}$ for code rate $R=2 / 3, \lambda=1 / 4$ and $k=1146$ bits. The spectrum has changed: some codewords disappeared and other codewords appeared with new distances, but all of them are largely bigger than 12 or 15 .

TABLE III. FIRST TERMS OF THE DISTANCE SPECTRUM FOR THE OPTIMIZED 3D TC WHERE $\mathrm{K}=1146 \mathrm{BITS}, \mathrm{R}=2 / 3$ AND $\Lambda=1 / 4$.

\begin{tabular}{|l|l|l|l|}
\hline Distance & 33 & 35 & 47 \\
\hline Multiplicity & 1 & 1 & 2 \\
\hline
\end{tabular}

\section{REDUCING THE CONVERGENCE LOSS OF 3- DIMENSIONAL TURBO CODES}

The use of the 3D 3GPP2 TC significantly increases the minimum Hamming distance, at the expense of a small increase in complexity and a loss of convergence at high error rates.

In this section, we analyse the convergence threshold of 3-dimensional turbo codes using the EXtrinsic Information Transfer (EXIT) chart, introduced by Stephan ten Brink [8], and we propose a method to reduce this loss of convergence.

\section{A. Determination of the Convergence Threshold of a Turbo Code}

To generate the EXIT chart of a TC, we have to consider the transfer characteristics of the extrinsic information for each Soft Input/Soft Output (SISO) decoder. Fig. 4 describes how the mutual information is measured in practice. After encoding, the information and parity bits $\mathrm{x}$ and $\mathrm{y}$ are transmitted over a noisy channel. The decoder receives the transmitted values at the channel output. Information bits $\mathrm{x}$ are also transmitted as $a$ priori knowledge, as if they were coming from the other decoder (lower input line to the decoder in Fig. 4). 


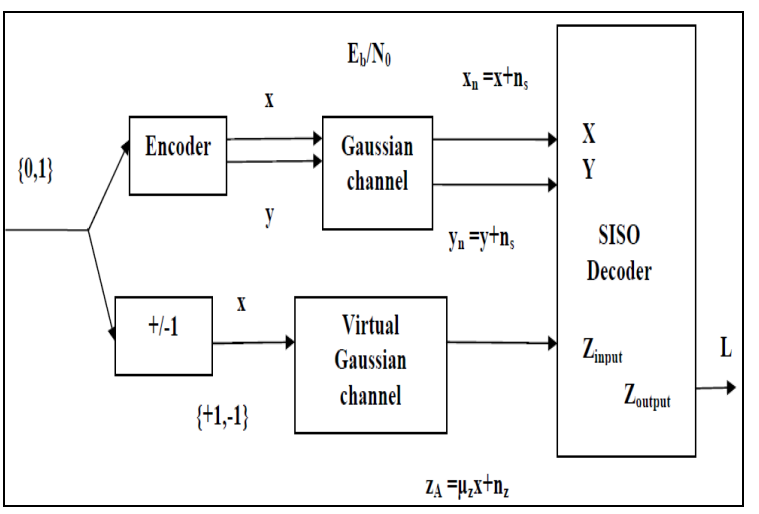

Figure 4. Measurement of mutual information at the component decoder level

The mutual information, between the $\mathrm{x}$ bits and the soft output values, $L$, of these bits after decoding can be computed from a large number $N$ of samples [9]:

$$
\begin{aligned}
I(L, X) & =1-E\left(1+\log _{2}\left(1+e^{-L}\right)\right) \\
& \approx \frac{1}{N} \sum_{k=1}^{N} \log _{2}\left(1+e^{-x_{k} L_{k}}\right)
\end{aligned}
$$

The convergence threshold of the turbo code is the minimum signal to noise ratio where the only intersection point is $(1,1)$.

When the signal to noise ratio is low, the curves have intersection points different from the point $(1,1)$ such as for $\mathrm{E}_{\mathrm{b}} / \mathrm{N}_{0}=1.4 \mathrm{~dB}$ in Fig. 5. Then, the iterative process starting with a zero average mutual information in entry cannot end in a perfect determination of the message.

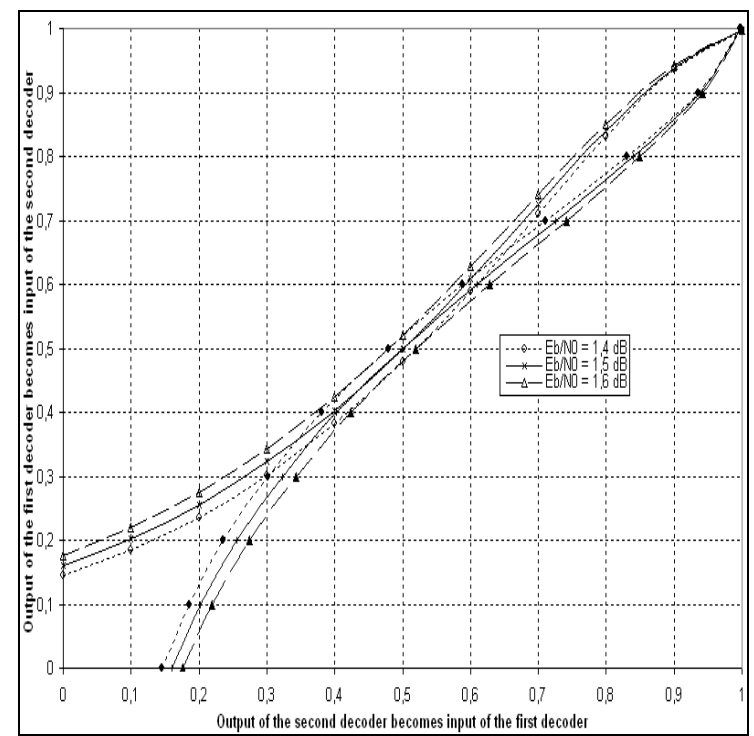

Figure 5.

EXIT chart of 8-state TC for different values of $\mathrm{E}_{\mathrm{b}} / \mathrm{N}_{0}$ at code rate $R=2 / 3$.

In the simulated example, the convergence threshold can be estimated around $1.49 \mathrm{~dB}$ for binary 8 -state turbo codes at code rate $R=2 / 3$.
B. Determination of the ConvergenceThreshold of 3-Dimensional Turbo Codes

In the case of 3D TC, the two 8-state SISO decoders exchange extrinsic information about the systematic part of the received codeword, like for classical turbo decoding. But both of them exchange also extrinsic information about the postencoded parity bits with the 4-state SISO predecoder. And we have to take into account in the EXIT chart that the extrinsic information about these parity bits is changing from an iteration to the other. Consequently, the curves of mutual information exchange between the two decoders change every iteration.

Fig.6 shows two EXIT charts of a 3D TC for different values of $E_{b} / N_{0}$. Note that, after the fourth iteration, the transfer characteristics remain almost unchanged.

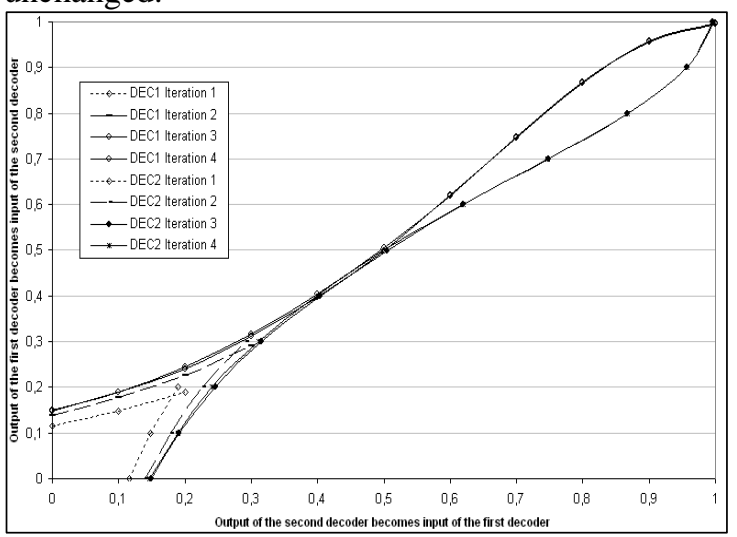

(a)

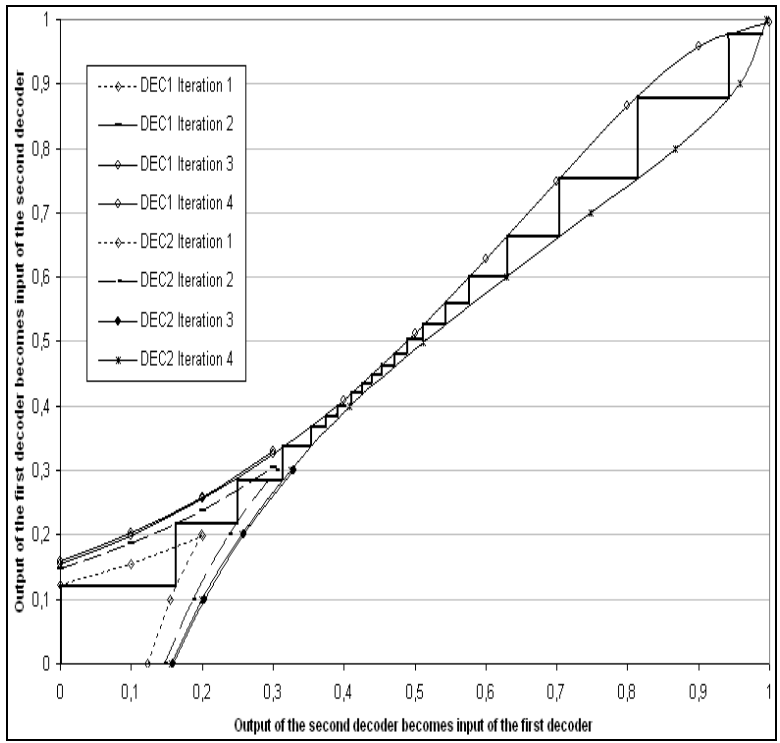

(b)

Figure 6. EXIT chart of 3D TC for at code rate $R=2 / 3$ and $\lambda=1 / 8$ :

a) $\mathrm{E}_{\mathrm{b}} / \mathrm{N}_{0}=1.5 \mathrm{~dB}$

b) $\mathrm{E}_{\mathrm{b}} / \mathrm{N}_{0}=1.55 \mathrm{~dB}$ 
For $\mathrm{E}_{\mathrm{b}} / \mathrm{N}_{0}=1.5 \mathrm{~dB}$ (see Fig. 6 a), the iterative process cannot converge to the point $(1,1)$ since the EXIT curves intersected before and the exchange of the extrinsic information between the two decoders about the systematic part of the received codeword is blocked. Whereas for $\mathrm{E}_{\mathrm{b}} / \mathrm{N}_{0}=1.55 \mathrm{~dB}$ (see Fig. 6 b), the tunnel between the EXIT curves is open, and the exchange of the extrinsic information continues along the iterations until we reach the intersection point $(1,1)$. These results were confirmed by the simulations of the code.

\section{3-Dimensional Turbo Codes Loss of Convergence}

The comparison of Fig. 5 and Fig. 6 b shows a loss of $0.06 \mathrm{~dB}$ for the convergence threshold of the 3 -dimensional turbo code compared with the original code, at code rate $R=2 / 3$ and $\lambda=1 / 8$. In Fig. 7 , we have plotted the FER performance of the 3D 3GPP2 TC to compare it with that of the 3GPP2 TC. As expected, we can observe a loss in convergence around $0.06 \mathrm{~dB}$ in the waterfall region. On the other hand, the low error rate performance is noticeably improved. The gain obtained for 1146 bits and $\mathrm{R}=2 / 3$ is about $0.9 \mathrm{~dB}$ at FER $=10^{-5}$.

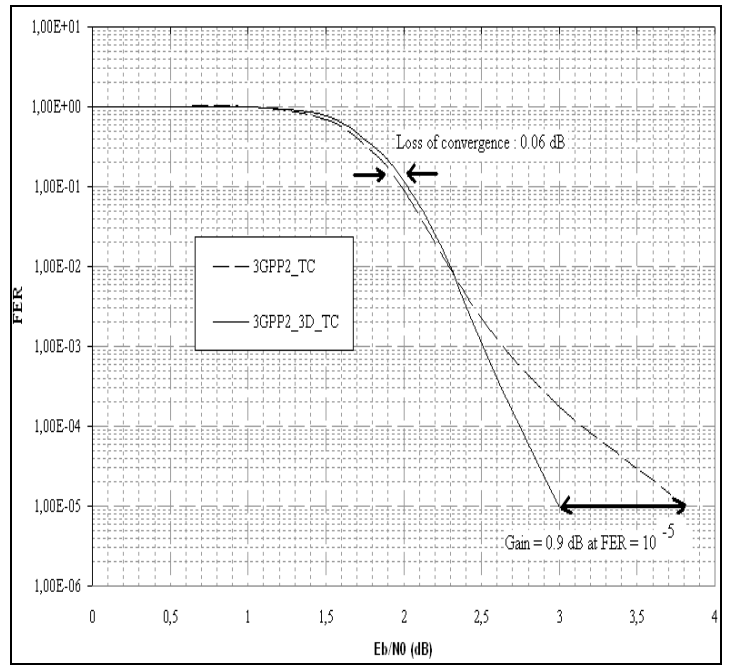

Figure 7. FER performance of the 3D 3GPP2 TC with $\lambda=1 / 8$ for $k=1146$ bits, $R=2 / 3$ and comparison with the

3GPP2 TC. All simulations used the MAP algorithm with 10 iterations.

If we use a larger permeability rate, the loss of convergence is more important. For instance, the observable loss of convergence for rate $2 / 3$ and $\lambda=1 / 4$ is $0.18 \mathrm{~dB}$. Even more, this loss of convergence increases when the code rate decreases. For example, the observable loss of convergence for rate $1 / 3$ and $\lambda=1 / 4$ is $0.26 \mathrm{~dB}$.

\section{Time Varying 3-Dimensional 3GPP2 Turbo Codes}

In order to reduce the loss of convergence of 3D TCs, we propose the adoption of a time-varying encoder as a rate- 1 post-encoder. Fig. 8 depicts the parity construction of the proposed encoder.

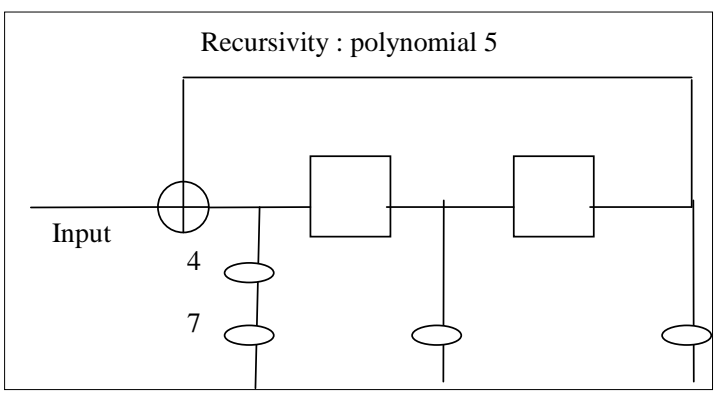

Figure $8 . \quad 4$-state post-encoder with time-varying parity construction $(5,4: 7)$. Redundancies 4 and 7 are alternated in time.

1) Time Varying Encoding: To simulate 3D TC using a 3GPP2 [5] interleaver, we have used the Recursive Systematic Convolutional (RSC) code $(5,4)$ as a post-encoder (see details in [6]). In order to obtain a Time Varying (TV) trellis, two redundancies $\mathrm{W}_{1}=4$ and $\mathrm{W}_{2}=7$ can be alternated in time instead of having only one [10]. The recursivity polynomial remains unchanged, that is 5. If we look at the trellis of this code, we can easily identify two all-zero sequences with exactly the same inputs and the same redundancies. So the decoder will not be able to distinguish between them and the distance of the code is only 2 , compared with 3 for the RSC code $(5,4)$.

In order to get closer to the code $(5,7)$ which distance is 5 , the idea is to replace periodically some redundancies $\mathrm{W}_{1}$ as described in Fig. 9. The replacement period is denoted by $L$.

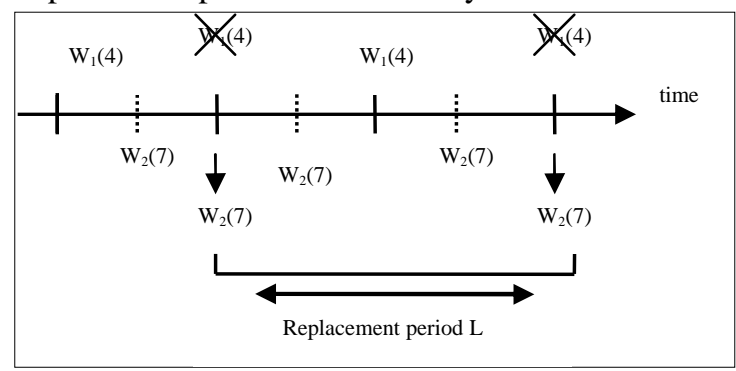

Figure 9. Modified time-varying post-encoding with a replacement period $L$

Then the challenge involves finding a value of $L$ which improves the performance of the 3D 3GPP2 TC, without loosing a lot in convergence at the first iteration. The code $(5,7)$ has a corresponding decoder which triples the number of errors of its input at the first step of the iterative process. The code $(5,4)$ has a corresponding decoder which only doubles the number of errors of its input at the first iteration: $B E R_{\text {out }}=2 B E R_{i n}$, where $B E R$ in is the channel error rate. Using a time varying post encoder increases the BER at its output. The BER at the first step is then expressed in the following way: $B E R_{\text {out }}=2 *\left(B E R_{\text {in }}+\xi\right)$, 
where the evolution of $\xi$, the additive error rate, according to $L$ is given in Fig. 10.

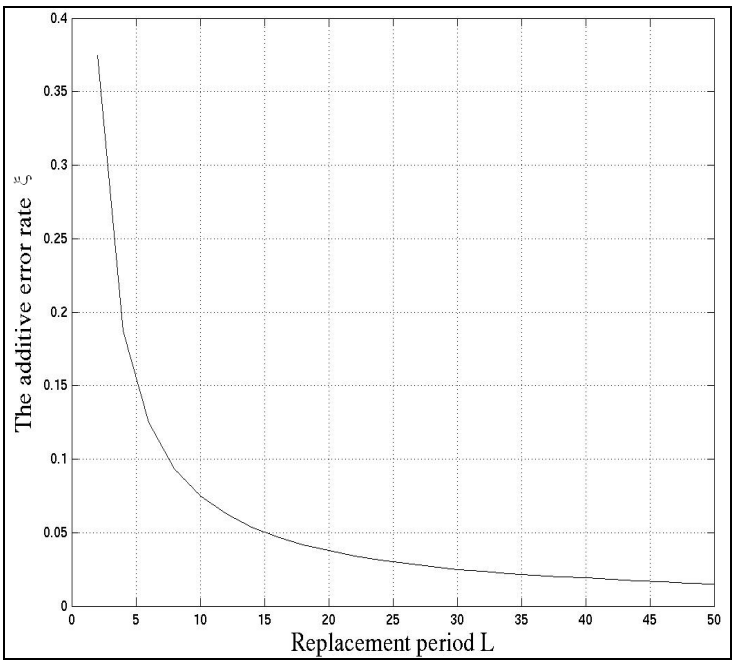

Figure 10. Estimation of the additive loss of convergence according to $L$ for $k=1530$ bits.

Fig. 10 shows that the loss of convergence is more significant at the first iteration for $L$ small, as the number of the redundancies $\mathrm{W}_{2}$ exceeds that of the redundancies $\mathrm{W}_{1}$. In this case, the time varying encoder is closer to the code $(5,7)$ which decoder triples the number of errors of its input. Then from the convergence point of view it is preferable to choose $L$ high. However, from the asymptotic performance point of view, it is better to choose $L$ small because the code $(5,7)$ has the higher distance, that is 5 . Thus, the optimal value of $L$ is a convergence/distance trade-off.

2) Time Varying Results for $k=1146$ bits: The FER performance of the time varying 3D 3GPP2 TC has been simulated with $\lambda=1 / 4$ and $\mathrm{L}=30$. Then one bit out of four is regularly picked from each of the parity streams starting with the first bit from each stream. For the post encoder, two redundancies $\mathrm{W}_{1}=4$ and $\mathrm{W}_{2}=7$ are alternated in time, but $\mathrm{W}_{1}$ is replaced by $\mathrm{W}_{2}$ for all the periods of $L=30$. Fig. 11 shows that the use of time varying 3-dimensional 3GPP2 TC reduced the loss of convergence by $50 \%$ from $0.18 \mathrm{~dB}$ to $0.09 \mathrm{~dB}$ for $k=1146$ bits at code rate $R=2 / 3$ and $\lambda=1 / 4$, compared with the 3D 3GPP2 TC. However, the asymptotic performance is not degraded.

On the other hand, the new convergence threshold estimated by the EXIT char method for the time varying 3D 3GPP2 turbo code, at code rate $R=2 / 3$ and $\lambda=1 / 4$, is $1.58 \mathrm{~dB}$ instead of $1.67 \mathrm{~dB}$ for the 3D 3GPP2 TC; which confirms the simulation results in Fig. 11. Therefore, the use of a time varying post encoder accelerates the convergence of the 3D turbo code.

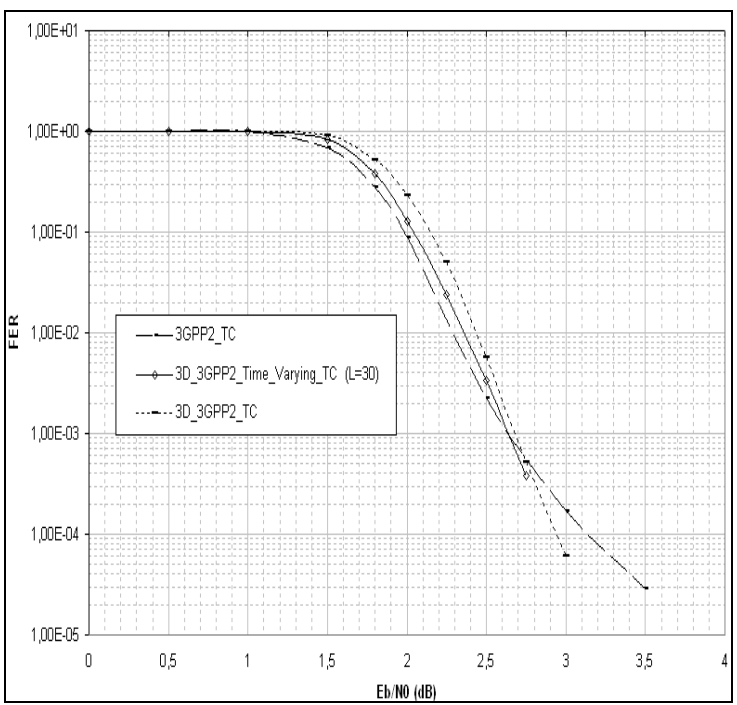

Figure 11. FER performance of the time varying 3D 3GPP2 TC with $\lambda=1 / 4$ for $k=1146$ bits, $R=2 / 3$ and comparison with the 3D 3GPP2 TC and 3GPP2 TC. All simulations used the MAP algorithm with 10 iterations.

\section{CONCLUSIONS}

The error rate curves of a turbo code can be divided into two regions: the waterfall region where the error rate decreases rapidly; and the region of error floor where a change in the curve slope appears when the code reaches its asymptotic gain. It is naturally desirable to have turbo codes which have waterfalls as closest as possible to the channel capacity and low floors of error.

First, the 3D TC significantly improves performance in the error floor region with respect to the 8-state classical turbo code. Then, in the case of the 3GPP2 code, a specific optimization of the permeability pattern allows to lower even more this floor. Finally, the time varying 3D TC reduces the loss of convergence of the 3D TC without degrading its asymptotic performance.

So, it is possible to build 3-Dimensional 3GPP2 turbo codes which have good performance in both regions. And this code structure is expected to reach a performance/complexity trade-off never yet attained.

\section{ACKNOWLEDGMENTS}

The authors would like to thank Professor C. Berrou for useful discussions and valuable suggestions.

\section{REFERENCES}

[1] C. Berrou, A. Glavieux, and P. Thitimajshima, "Near Shannon limit error-correcting coding and decoding: turbocodes," IEEE International Conference on Communications. Geneva, Switzerland, May 1993, pp. 1064-1070.

[2] Third Generation Partnership Project (3GPP) Technical Specification Group, "Multiplexing and channel coding (FDD)," June 1999, TS 25.212, v2.0.0. 
[3] DVB,"Interaction channel for satellite distribution systems, " December 2000, ETSI EN 301 790, v. 1.2.2.

[4] DVB, "Interaction channel for digital terrestrial television," Mars 2001, ETSI EN 301 958, v. 1.1.1.

[5] Third Generation Partnership Project 2 (3GPP2), "Physical layer standard for cdma2000 spread spectrum systems, Release D," Feb. 2004, 3GPP2 C.S0002-D, Version 1.0.

[6] C. Berrou, A. Graell i Amat, Y. Ould-CheikhMouhamedou, C. Douillard, and Y. Saouter, "Adding a rate-1 third dimension to turbo codes," in Proc. IEEE Inform. Theory Workshop, Lake Tahoe, CA, Sep. 2007, pp. $156-161$.

[7] R. Garello and A. Casado, "The All-Zero Iterative Decoding Algorithm for Turbo Code Minimum Distance Computation," IEEE International Conference on Communications, Paris, France, pp. 361-364, June 2004.

[8] S. Ten Brink. "Convergence of iterative decoding," Electronics Letters, 35, June 1999, pp. 806-808.

[9] J. Hagenauer, "The EXIT chart Introduction to extrinsic information transfer in iterative processing," in Proc. Eur. Signal Process. Conf., Vienna, Austria, Sep. 2004, pp. $1541-1548$.

[10] C. Berrou, A. Graell i Amat and Y. Ould-CheikhMouhamedou, "About rate-1 codes as inner codes," 5th International Symposium on Turbo Codes and Related Topics, Lausanne, Switzerland, Sep. 2008. 